\title{
Role of octanoic and decanoic acids in the control of seizures
}

\author{
M A SILLS, W I FORSYTHE, AND D HAIDUKEWYCH
}

General Infirmary and St James's University Hospital, Leeds, and Epilepsy Centre of Michigan, Detroit, United States of America

SUMMARY Octanoic and decanoic acid, the major constituents of the Medium Chain Triglyceride (MCT) Emulsion diet, have been detected in appreciable quantities in the peripheral blood of children with intractable seizures treated with the MCT diet. Serum concentrations of these acids as well as $\beta$ hydroxybutyrate and acetoacetate rose as the diet was introduced and on full diet showed pronounced diurnal variation and low concentrations in the morning. No correlation between octanoic and decanoic acid concentrations and control of seizures was established, but further studies with octanoic and decanoic acid using animal models are necessary to assess the role of these acids and of control of seizures.

In a recent review Kupferberg reported that experimental animals were afforded optimal protection against seizures induced by pentylenetrazol by $\mathrm{C}_{8}-\mathrm{C}_{10}$ carboxylic acids. ${ }^{1}$

The Medium Chain Triglyceride (MCT) diet is a form of neutral lipid triglyceride containing fatty acid molecules with a carbon chain length of 6 to 12 , the major constituents being octanoic acid $81 \%$ and decanoic acid $15 \%$ (Table 1 ). These acids are lipid soluble and are absorbed rapidly from the gastrointestinal tract through the portal circulation to the liver, where it was assumed that they were metabolised to carbon dioxide, ketone bodies, and long chain fatty acids with little escape into the systemic circulation. Linscheer et al were able, however, to measure octanoic acid concentrations in the blood of normal patients and patients with cirrhosis after duodenal infusions and were surprised to find small amounts in the blood of the normal group. ${ }^{2}$ From their data we have calculated the mean serum concentration to be about $6 \mu \mathrm{g} / \mathrm{ml}$.

Recently, we reported significant concentrations of both octanoic and decanoic acids in the serum of

Table 1 Constituents of the liquidised MCT diet

\begin{tabular}{lr}
\hline Acid & $\%$ \\
\hline Hexanoic $\left(\mathrm{C}_{6}\right)$ & $1 \cdot 1$ \\
Octanoic $\left(\mathrm{C}_{8}\right)$ & $81 \cdot 1$ \\
Decanoic $\left(\mathrm{C}_{10}\right)$ & $15 \cdot 7$ \\
Dodecanoic $\left(\mathrm{C}_{12}\right)$ & $2 \cdot 1$ \\
\hline
\end{tabular}

children receiving MCT Emulsion for the control of drug resistant seizures. ${ }^{3}$

It seems reasonable to suggest that as these fatty acids occur in appreciable amounts in the peripheral blood, especially octanoic acid, they could have an effect on the central nervous system. It is known that a direct isomer of octanoic acid-namely, valproic acid-has anticonvulsant properties. The results of our recent study of children receiving the MCT Emulsion diet has shown that four children with drug resistant seizures have remained free of seizures for six months to two years on diet alone. ${ }^{4}$ This suggested that the diet contained agents that acted directly or indirectly on the control of seizures. We thought it worth while to investigate the role of octanoic, decanoic, $\beta$ hydroxybutyrate, acetoacetate, and alanine fatty acids in children taking the MCT Emulsion diet.

\section{Patients and methods}

Octanoic and decanoic acids were assayed by gas liquid chromatography as previously described. ${ }^{3}$ Blood samples were collected in heparinised tubes and the separated plasma stored at $4^{\circ} \mathrm{C}$. All samples were analysed blind in Detroit without the analyst being aware of individual clinical details. Initially, random morning samples were obtained before beginning treatment, during initial starvation, and on full diet. More recently we assayed concentrations at fixed times during a 24 hour period in children who were receiving $50-60 \%$ of their energy 
requirements from MCT Emulsion: (i) at four sampling times in eight children and (ii) at five sampling times in a further nine children. In these children we estimated serum concentrations of $\beta$ hydroxybutyrate, acetoacetate, and alanine. Two children were included in both studies and a child in the second study had an extra sample taken at $0400 \mathrm{~h}$.

\section{Results}

The initial morning samples produced undetectable concentrations of octanoic and decanoic acids in five children before treatment and during fasting and in

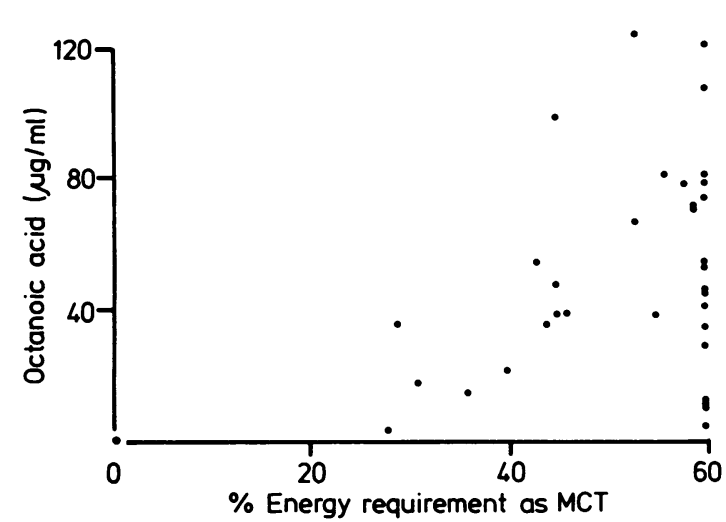

Fig. 1 Relation between serum concentrations of octanoic acid and increasing amounts of MCT diet.

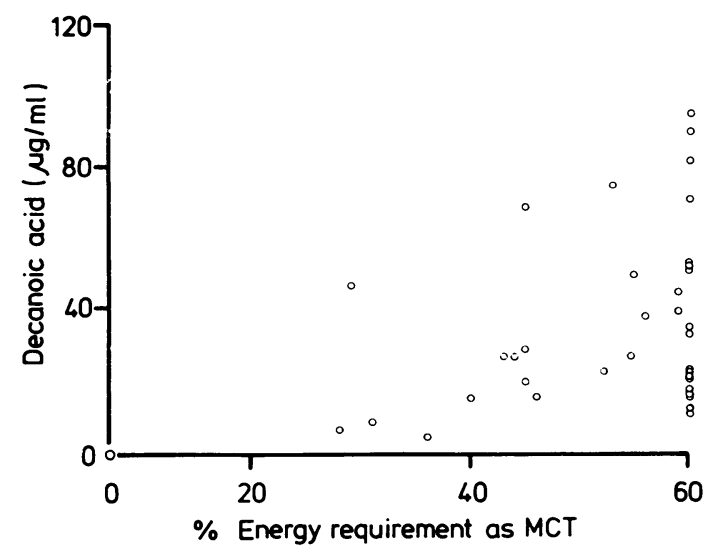

Fig. 2 Relation between serum concentrations of decanoic acid and increasing amounts of MCT diet. two children after withdrawal of treatment. In children on the MCT Emulsion diet fatty acid concentrations seemed to rise as the amount was increased until $60 \%$ of the energy requirements was supplied by the diet (Figs. 1 and 2). Concentrations of octanoic acid were higher than those of decanoic acid. The concentrations obtained compared with blood concentrations for other anticonvulsants-for example, sodium valproate and clonazepam. As there was a wide scatter of results on 'full diet' we decided to sample at fixed times during the 24 hours.

The first study of 24 hour profiles in eight children at $0800,1200,1600$, and $2000 \mathrm{~h}$ showed a pronounced diurnal variation of concentrations of octanoic and decanoic acids on 50-60\% MCT (Fig. 3 ). In the second study of 24 hour profiles in nine children at five sampling times-namely, 1200, $1600,2000,2400$, and 0800 h the next day - when the mean values for octanoic and decanoic acids were plotted the same trend was repeated (Fig. 4). A similar diurnal variation was seen with concentrations of $\beta$ hydroxybutyrate and acetoacetate but not with alanine (Fig. 4).

In one patient the 24 hour profiles at six sampling times-namely, 1200, 1600, 2000, 2400, 0400, and $0800 \mathrm{~h}$ the next day - showed that the most rapid fall in octanoic and decanoic acids, $\beta$ hydroxybutyrate, and acetoacetate occurred between 0400 and $0800 \mathrm{~h}$ (Fig. 5).

Clinical details for the children involved in these

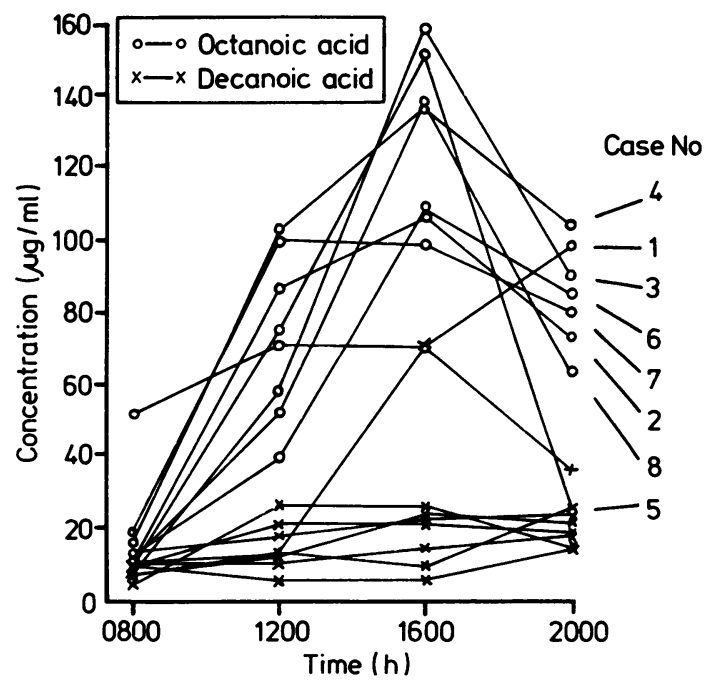

Fig. 3 Twenty four hour serum profles of concentrations of octanoic and decanoic acids in eight children at four sampling times. 


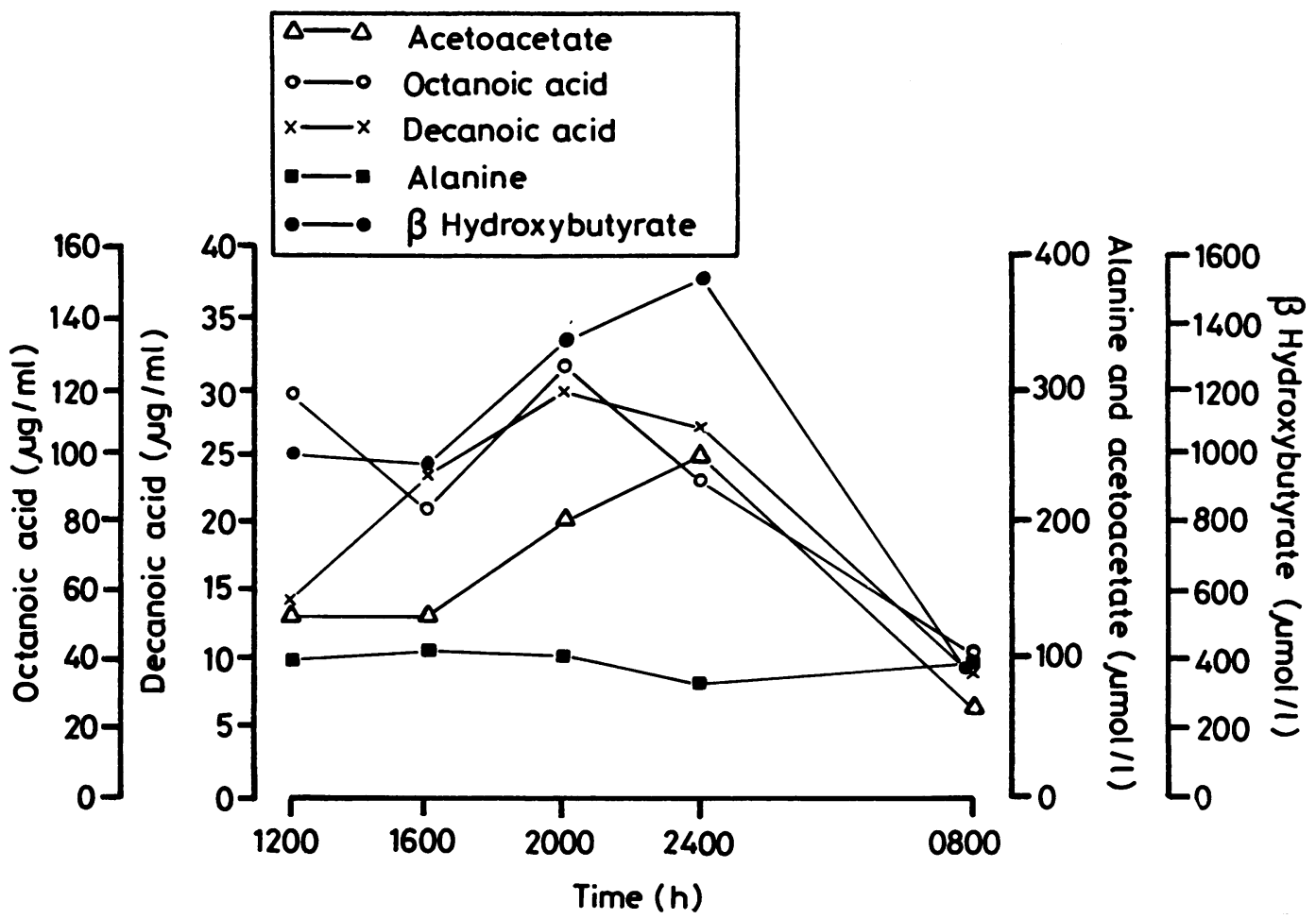

Fig. 4 Mean serum concentrations of octanoic and decanoic acids, $\beta$ hydroxybutyrate, acetoacetate, and alanine in nine children at five sampling times.

two profile studies as regards age at time of study, duration of time since beginning the MCT Emulsion diet, seizure type, and effectiveness of control are shown in Tables 2 and 3 along with the maximum serum concentrations of octanoic and decanoic acids for each child during each profile. Effectiveness of control is assessed according to our reported results. ${ }^{4}$

\section{Discussion}

In our initial studies where serum samples were taken at unstandardised times we found the concentrations of octanoic and decanoic acids to vary widely, but a gradual increase in concentrations while becoming established on the diet was suggested. We considered poor compliance a probable cause but wished to check if concentrations fluctuated during the day. We were surprised to find such a wide fluctuation in concentrations in the first profile series, with low morning and high afternoon and evening concentrations common to all patients even after long periods on the diet. This diurnal variation would suggest that octanoic and decanoic acids in MCT are cleared from the blood and presumably metabolised very quickly, indicating that continuous or frequent administration of small doses of MCT would lead to more persistent concentrations, and this might be reflected in a similar change for $\beta$ hydroxybutyrate and acetoacetate, as our second profile series shows a similar diurnal variation for these ketogenic acids.

Although a relation between control of seizures and $\beta$ hydroxybutyrate concentrations has been suggested previously, we did not achieve the same high concentrations as Huttenlocher, but we had success in reducing seizures. ${ }^{5}$ It may be that optimal control of seizures with MCT Emulsion could be achieved with random $\beta$ hydroxybutyrate concentrations lower than those suggested by Huttenlocher. ${ }^{5}$

The low morning concentrations were of interest, and we note that low morning ketone concentrations have been reported in children on the ketogenic diet and that this was the opposite to what was seen in normal children. ${ }^{67}$ The low concentrations might relate to our previous observation that nocturnal seizures were not so readily suppressed on the diet. ${ }^{4}$ It is possible that these observations tell us how the 
diet should be used rather than how it works. MCT Emulsion given at night should improve morning fatty acid concentrations and could reduce the tendency to seizures at the time.

The 15 children involved in these profiles (Tables 2 and 3) had a varied degree of control of seizures and varied types of seizures. It is thus difficult to draw conclusions about the relation between control of seizures and serum concentrations, but all children have optimum concentrations of octanoic acid in excess of $90 \mu \mathrm{g} / \mathrm{ml}$ and maximum concentrations of decanoic acid in excess of $8 \mu \mathrm{g} / \mathrm{ml}$. There was no clear association, however, between concentrations of these acids and control of seizures, seizure type, age of child, or duration on diet in this small series. A similar lack of correlation between serum concentrations and control of seizures has been found with sodium valproate and clonazepam.

We have therefore not been able to show any definite relation between fatty acid concentrations and control of seizures, but it would not be unreasonable to suppose that octanoic and decanoic

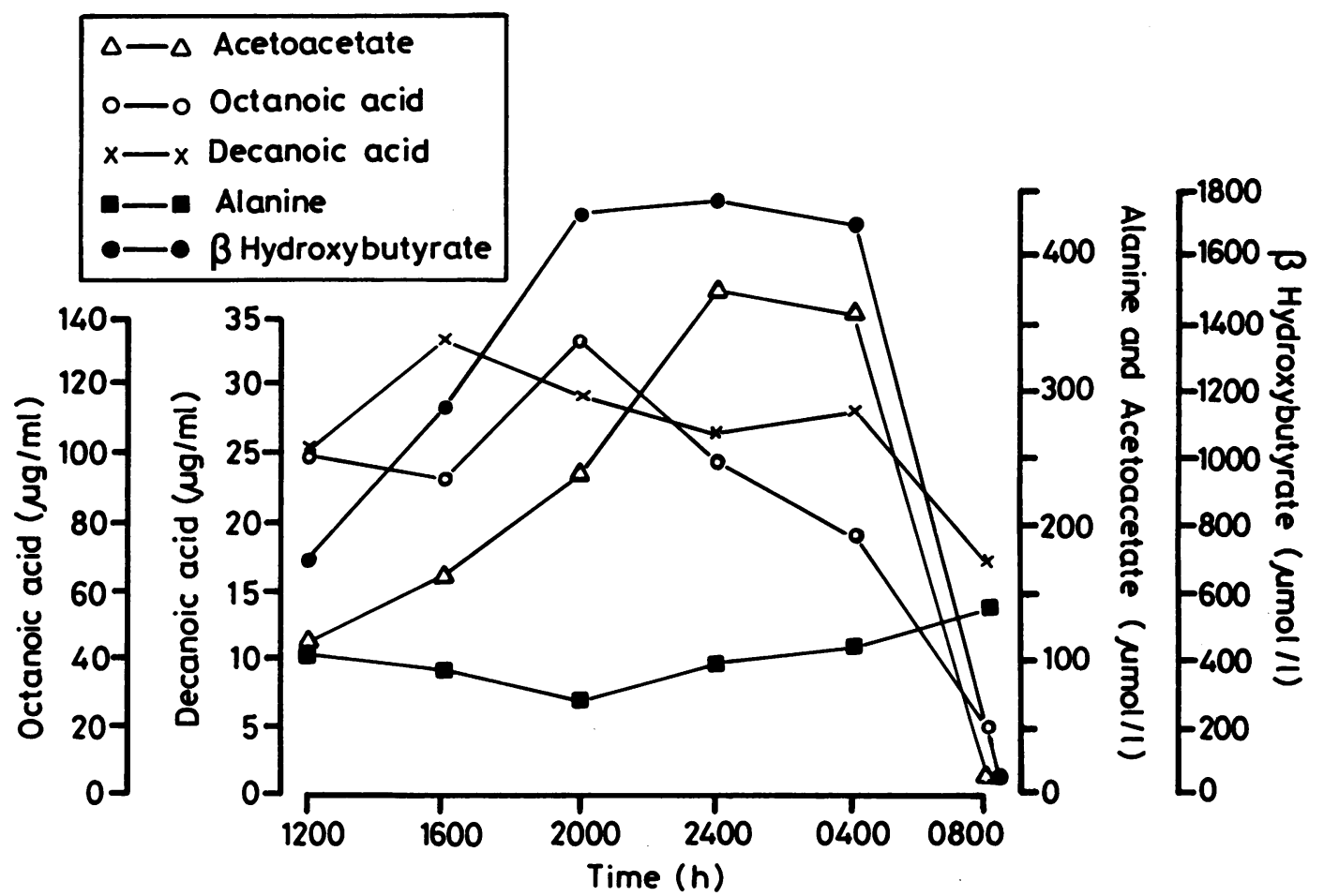

Fig. 5 Twenty four hour serum profiles of concentrations of octanoic and decanoic acids, $\beta$ hydroxybutyrate, acetoacetate, and alanine in a patient on full MCT diet at six sampling times.

Table 2 Clinical information for the eight patients in Figure 3

\begin{tabular}{|c|c|c|c|c|c|}
\hline \multirow[t]{2}{*}{ Case no } & \multirow[t]{2}{*}{ Age (years) } & \multirow[t]{2}{*}{ Diagnosis } & \multirow[t]{2}{*}{ Seizure control } & \multicolumn{2}{|c|}{ Maximum concentration $(\mu \mathrm{g} / \mathrm{ml})$} \\
\hline & & & & $\begin{array}{l}\text { Octanoic } \\
\text { acid }\end{array}$ & $\begin{array}{l}\text { Decanoic } \\
\text { acid }\end{array}$ \\
\hline 1 & 9 & Grand mal & Good & $98 \cdot 2$ & $12 \cdot 2$ \\
\hline 2 & 3 & Astatic myoclonic & Complete & $104 \cdot 7$ & $24 \cdot 5$ \\
\hline 3 & 10 & Grand mal & Good & $157 \cdot 7$ & $22 \cdot 6$ \\
\hline 4 & 5 & Astatic myoclonic & Complete & $134 \cdot 9$ & $22 \cdot 4$ \\
\hline 5 & 4 & Astatic myoclonic & Excellent & 149.4 & $26 \cdot 6$ \\
\hline 6 & 11 & Petit mal & Excellent & $109 \cdot 5$ & $15 \cdot 8$ \\
\hline 7 & 13 & Temporal lobe & Good & $101 \cdot 7$ & $20 \cdot 6$ \\
\hline 8 & 13 & Petit mal & Excellent & 137.7 & $70 \cdot 1$ \\
\hline
\end{tabular}

Seizure control: Complete $=100 \%$ reduction in seizures; Excellent $=90-100 \%$ reduction; Good $=50-90 \%$ reduction; and Poor $=<50 \%$ reduction. 
Table 3 Biochemical data for the nine children in Figure 4

\begin{tabular}{|c|c|c|c|c|c|}
\hline \multirow[t]{2}{*}{ C'ase no } & \multirow[t]{2}{*}{ Age (years) } & \multirow[t]{2}{*}{ Diagnosis } & \multirow[t]{2}{*}{ Seizure control } & \multicolumn{2}{|c|}{ Maximum concentration $(\mu \mathrm{g} / \mathrm{ml})$} \\
\hline & & & & $\begin{array}{l}\text { Octanoic } \\
\text { acid }\end{array}$ & $\begin{array}{l}\text { Decanoic } \\
\text { acid }\end{array}$ \\
\hline 1 & 12 & Grand mal & Good & $136 \cdot 2$ & $34 \cdot 4$ \\
\hline 2 & 2 & Temporal lobe & Complete & $124 \cdot 0$ & $29 \cdot 4$ \\
\hline 3 & 7 & Temporal lobe & Poor & $151 \cdot()$ & $33 \cdot 6$ \\
\hline 4 & 14 & Grand mal & Good & $123 \cdot 3$ & 8.0 \\
\hline 5 & 11 & Petit mal & Excellent & $123 \cdot 5$ & $15 \cdot 2$ \\
\hline 6 & 10 & Grand mal & Good & $175 \cdot 1$ & $35 \cdot 5$ \\
\hline 7 & 5 & Astatic myoclonic & Complete & 258.9 & $80 \cdot 6$ \\
\hline 8 & 13 & Astatic myoclonic & Excellent & $100 \cdot 6$ & $23 \cdot 2$ \\
\hline 9 & 8 & Temporal lobe & Poor & Samples $n$ & \\
\hline
\end{tabular}

Seizure control: Complete $=10(0 \%$ reduction in scizures: Excellent $=90-100 \%$ reduction: Good $=50-90 \%$ reduction: and Poor $=<50 \%$ reduction

* Results for acetoacetate, alanine, and $\beta$ hydroxybutyrate are available for this patient. however, and are included in Figure 4

acids could have some influence on seizure discharge, and we think this justifies further investigation.

\section{Conclusions}

Our biochemical investigations led us to the following conclusions.

(1) Octanoic and decanoic acids occur in the peripheral blood in appreciable amounts in children receiving MCT Emulsion and are therefore not fully metabolised in the liver.

(2) There was a definite trend for concentrations of octanoic and decanoic acids to rise with an increasing amount of MCT Emulsion.

(3) The concentrations of these acids vary widely during the day and fall rapidly after the last evening dose of MCT, suggesting a short half life.

(4) Similar diurnal variations of $\beta$ hydroxybutyrate and acetoacetate were seen and these may follow rather than precede the changes in octanoic and decanoic acids.

\section{References}

' Kupferberg HG. Sodium valproate. Adv Neurol 1980;27:64354.

2 Linscheer WG, Patterson JF, Moore EW, Clermont RJ, Robins SJ, Chalmers TC. Medium and long chain fat absorption in patients with cirrhosis. J Clin Invest 1966;45:1317-25.

3 Haidukewych D. Forsythe WI. Sills MA. Monitoring octanoic and decanoic acids in plasma from children with intractable epilepsy treated with medium chain triglyceride dict. Clin Chem 1982:28:642-5.

${ }^{4}$ Sills MA, Forsythe WI, Haidukewych D, MacDonald A. Robinson M. The medium chain triglyceride diet (MCT) and intractable epilepsy. Arch Dis Child 1986;61:1168-72.

5 Huttenlocher PR. Ketonacmia and seizures: metabolic and anticonvulsant effects of two ketogenic diets in childhood epilepsy. Pediatr Res 1976:10:536-40.

- Clark BJ, House FM. Medium chain triglyceride oil ketogenic diets in the treatment of childhood epilepsy. Journal of Human Nutrition 1978:32:111-6.

${ }^{7}$ Schwartz RH, Aynesley-Green A. Bower BP. Clinical and metabolic aspects of the ketogenic diet. Research and Clinical Forums 1980;2:63-73.

Correspondence to Dr W I Forsythe. Clarendon Wing. The General Infirmary at Leeds, Belmont Grove, Leeds LS2 9NS, England.

Received 7 July 1986 